\title{
Effect of metabolic control on interferon- $\gamma$ and interleukin-10 production by peripheral blood mononuclear cells from type 1 and type 2 diabetic patients
}

\author{
M.C. Foss-Freitas ${ }^{1}$, \\ N.T. Foss' ${ }^{2}$, \\ E.A. Donadi ${ }^{3}$ \\ and M.C. Foss ${ }^{1}$
}

\author{
${ }^{1}$ Divisão de Endocrinologia e Metabolismo, ${ }^{2}$ Divisão de Dermatologia, \\ ${ }^{3}$ Divisão de Imunologia Clínica, Departamento de Clínica Médica, \\ Faculdade de Medicina de Ribeirão Preto, Universidade de São Paulo, \\ Ribeirão Preto, SP, Brasil
}

\section{Correspondence}

M.C. Foss

Departamento de Clínica Médica FMRP, USP

Av. Bandeirantes, 3900

14049-900 Ribeirão Preto, SP

Brasil

Fax: +55-16-3633-6695

E-mail: mcfoss@fmrp.usp.br

Publication supported by FAPESP. $\ldots \ldots \ldots \ldots \ldots \ldots$

Received July 1, 2006 Accepted February 27, 2007

\section{Abstract}

The objective of the present study was to evaluate the production of cytokines, interferon- $\gamma$ (INF- $\gamma$ ) and interleukin-10 (IL-10), in cultures of peripheral blood mononuclear cells (PBMC) from type 1 and type 2 diabetic patients and to correlate it with inadequate and adequate metabolic control. We studied 11 type 1 and 13 type 2 diabetic patients and 21 healthy individuals divided into two groups $(\mathrm{N}=11$ and 10$)$ paired by sex and age with type 1 and type 2 diabetic patients. The PBMC cultures were stimulated with concanavalin-A to measure INF- $\gamma$ and IL-10 supernatant concentration by ELISA. For patients with inadequate metabolic control, the cultures were performed on the first day of hospitalization and again after intensive treatment to achieve adequate control. INF- $\gamma$ levels in the supernatants of type 1 diabetic patient cultures were higher compared to type 2 diabetic patients with adequate metabolic control $(\mathrm{P}<0.001)$. Additionally, INF- $\gamma$ and IL-10 tended to increase the liberation of PBMC from type 1 and 2 diabetic patients with adequate metabolic control $(\mathrm{P}=0.009$ and 0.09 , respectively). The increased levels of INF- $\gamma$ and IL-10 released from PBMC of type 1 and 2 diabetic patients with adequate metabolic control suggest that diabetic control improves the capacity of activation and maintenance of the immune response, reducing the susceptibility to infections.

\section{Introduction}

Cytokines are produced in response to infection with microorganisms or other antigens and are known to be important immune modulators in host defense against aggressors (1). They also regulate cell functions in
Key words

- Type 1 diabetes mellitus

- Type 2 diabetes mellitus

- Cytokines

- Interferon- $\gamma$

- Interleukin-10

- Metabolic control other systems, being implicated in the development of chronic complications of diabetes mellitus, which involve neurological and vascular processes $(2,3)$. Chronic hyperglycemia, more specifically due to the increase of glycated proteins, can stimulate the production of cytokines (2) involved in the acti- 
vation of the immune system. The duration and metabolic control of diabetes mellitus seem to be important for the onset and progression of these complications (4-6). Besides vascular and neurological complications, a high susceptibility to infection has been described in diabetic patients (7-14), which may be caused by several defects of the immunological defense system.

Impairment of polymorphonuclear leukocyte phagocytosis and reduction in granulocyte phagocytic capacity have been reported with the increase of plasma glucose concentration in diabetic patients, and these abnormalities are reversed after insulin therapy (15-20). The most dramatic defect that occurs in diabetes mellitus is related to abnormalities of $\mathrm{T}$ cell function $(21,22)$. The reason for these alterations in the immune cellular behavior of diabetic patients is still undefined, with few studies of lymphocyte proliferation and none regarding the influence of metabolic control in diabetic patients.

Therefore, it seems important to determine the immune cellular behavior of diabetic patients focusing on $\mathrm{T}$ cell functionality with the objective to study the mechanisms that determine high susceptibility to infections in these patients. A high frequency of cutaneous dermatophytosis has been observed (23) in diabetic patients under inadequate metabolic control, suggesting that metabolic control is an important tool in the defense against these infections. In a previous study, we observed increased proliferation capacity of type 1 diabetic T lymphocytes (24) and a tendency towards increased tumor necrosis factor- $\alpha$ and interleukin- 6 (IL-6) levels after metabolic control of type 1 and 2 diabetic patients, suggesting that the control of diabetes improves the capacity of activation and maintenance of the pro-inflammatory immune response (25). On this basis, in the present investigation we studied the production of the cytokines interferon- $\gamma$ (IFN- $\gamma$ ) and IL-10 in cultures of peripheral blood mononuclear cells (PBMC) from type 1 and type 2 diabetic patients in order to correlate it with inadequate and adequate metabolic control.

\section{Subjects and Methods}

Twenty-four diabetic patients were selected from the Outpatient Clinics of the University Hospital of the Medical School of Ribeirão Preto, USP, Ribeirão Preto, SP, Brazil (Table 1). We also studied 21 healthy individuals paired for sex, age and body mass index with type 1 and type 2 diabetic patients. The patients presented inadequate metabolic control (fasting glucose higher than $200 \mathrm{mg} / \mathrm{dL}$ and glycated hemoglobin higher than $11 \%$ ), but did not present any infectious disease or were using any drugs that might interfere with the results. The patients were hospitalized for 2-3 weeks to obtain adequate metabolic control by using an intensive protocol with capillary blood glucose measurements at $7,11,17$, and $23 \mathrm{~h}$, and regular administration of insulin injections until a reduction of at least $100 \mathrm{mg} / \mathrm{dL}$ in mean daily glycemic level was reached. In addition to the neurological examination, the presence of microvascular complications was evaluated by ophthalmologic examination and urine protein measurement and the presence of macrovascular complications by clinical and electrocardiographic evaluation. BMI was calculated as weight $(\mathrm{kg}) /$ height $(\mathrm{m})^{2}$. The protocol was approved by the Ethics Committee of the University Hospital (FMRP, USP) and the volunteers were carefully informed about the nature, purpose and possible risks of the study before giving written informed consent to participate.

Blood samples were obtained on the first and last days of hospitalization. Further blood samples were obtained for the measurement of glycated hemoglobin (Labtest Diagnóstica, Lagoa Santa, MG, Brazil) (20). PBMC were isolated by gradient density using Ficoll-Hypaque $^{\circledR}$ (26). PBMC at the concen- 
tration of $2.5 \times 10^{6}$ cells $/ \mathrm{mL}$ were cultivated in triplicate in the presence or absence of concanavalin-A. After a 72 -h culture at $37^{\circ} \mathrm{C}$ in a humid environment with approximately $5 \% \mathrm{CO}_{2}$, the supernatant was collected for cytokine measurements. IL-10 and IFN- $\gamma$ were assayed in cell culture supernatants by ELISA. Monoclonal anti-human IL-10 and IFN- $\gamma$ antibody (Pharmingen International, San Diego, CA, USA) was used as capture antibody and biotinylated anti-human IL-10 and IFN- $\gamma$ (Pharmingen) as the detecting antibody. Binding was determined with peroxidase-labeled streptavidin (DAKO, Glostrup, Denmark) and O-phenylenediamine$2 \mathrm{HCl} /$ substrate (Sigma, St. Louis, MO, USA). The intra- and interassay variation was below $10 \%$. The ELISA detection limits were $0.07 \mathrm{pg} / \mathrm{mL}$ for INF- $\gamma$ and $0.03 \mathrm{pg} / \mathrm{mL}$ for IL-10.

Data are reported as median, mean and standard deviation. The GraphPad Prism program (San Diego, CA, USA) was used for statistical analysis. The Kruskal-Wallis test was used to analyze differences between the three groups (type 1 and type 2 patients, and controls), and the Wilcoxon test was used to compare the diabetic groups before and after metabolic control. The Spearman test was used to calculate all correlations. A P value $<0.05$ was considered to be significant.

\section{Results}

The four groups studied, i.e., type 1 and type 2 diabetic patients and healthy individuals, were statistically similar in age, sex and body mass index (Table 1). The patients did not present chronic macro- or microvascular or neurological complications. The metabolic control evaluated by the daily mean glycemic profile during the hospitalization period showed a significant improvement with treatment between the first and last days of hospitalization in type 1 and type 2 diabetic patients $(\mathrm{P}=0.0002$ and $\mathrm{P}=0.0001$, respectively; Figure 1).
IFN- $\gamma$ levels were higher in the supernatants of stimulated cultures from type 1 diabetic patients compared to type 2 diabetic patients, both with adequate metabolic control $(\mathrm{P}<0.001$; Figure 2$)$. Additionally, the improvement of metabolic control increased the concentrations of IFN- $\gamma$ in the stimulated culture supernatants of type 1 diabetic patients when compared to type 1 diabetic patients with inadequate metabolic control $(\mathrm{P}=0.009$; Figure 2). However, metabolic control did not influence the IFN- $\gamma$ production by PBMC of type 2 diabetic patients. In contrast, IL-10 levels were similar in the supernatants of cultures from type 1 and 2

Table 1. Characteristics of type 1 and type 2 diabetic patients and healthy individuals paired with type 1 and type 2 patients.

\begin{tabular}{lcccc}
\hline & $\begin{array}{c}\text { Type 1 } \\
\text { diabetic } \\
\text { patients } \\
(\mathrm{N}=11)\end{array}$ & $\begin{array}{c}\text { Type 2 } \\
\text { diabetic } \\
\text { patients } \\
(\mathrm{N}=13)\end{array}$ & $\begin{array}{c}\text { Control } \\
\text { paired } \\
\text { with type 1 } \\
(\mathrm{N}=11)\end{array}$ & $\begin{array}{c}\text { Control } \\
\text { paired } \\
\text { with type 2 } \\
(\mathrm{N}=10)\end{array}$ \\
\hline Age (years) & $22.7 \pm 7.1$ & $49.7 \pm 18.2$ & $24.5 \pm 7.1$ & $39.5 \pm 15.8$ \\
Gender (female/male) & $6 / 5$ & $3 / 10$ & $8 / 3$ & $2 / 8$ \\
Body mass index $\left(\mathrm{kg} / \mathrm{m}^{2}\right)$ & $23.1 \pm 3.8$ & $28.5 \pm 8$ & $21.4 \pm 2.8$ & $24.7 \pm 9.1$ \\
$\begin{array}{l}\text { Glycated hemoglobin }(\%) \\
\text { Duration of diabetes } \\
\text { mellitus (years) }\end{array}$ & $11.7 \pm 2$ & $12 \pm 2.3$ & $5.7 \pm 0.9$ & $5.5 \pm 1.6$ \\
& $9.6 \pm 5.8$ & $10.2 \pm 7$ & - & - \\
\hline
\end{tabular}

Data are reported as mean \pm SD

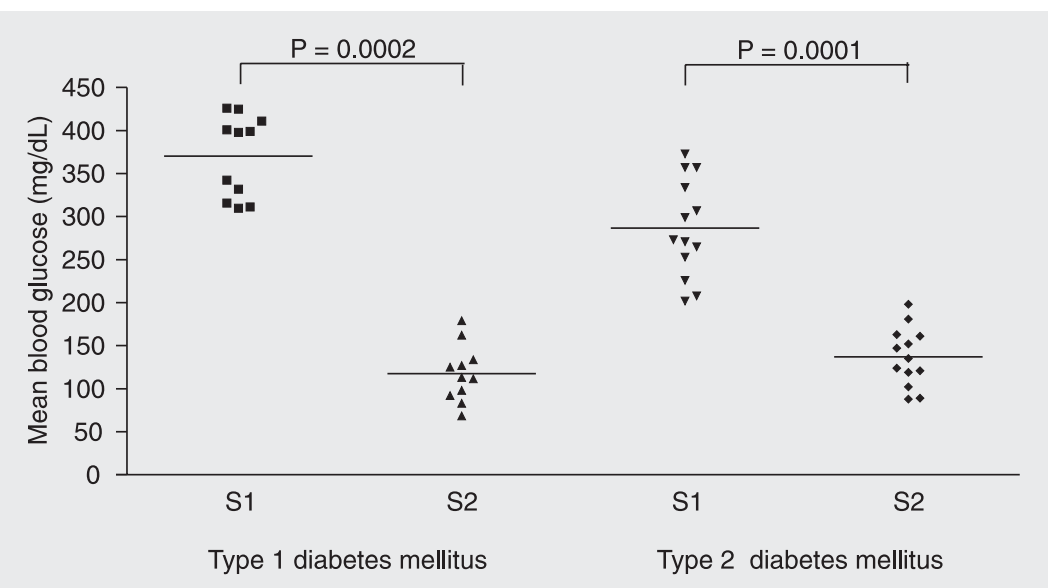

Figure 1. Daily mean glucose levels of type 1 and type 2 diabetic patients on the first (sample 1, S1) and last (sample 2, S2) days of hospitalization. There was a significant reduction in the daily mean blood glucose levels in both type 1 and 2 diabetic groups. The Student $t$-test was used. The horizontal lines indicate the mean for each group. 
diabetic patients with inadequate and adequate metabolic control compared with their respective paired control groups. However, this cytokine showed a nonsignificant increase in the supernatants of type 2 diabetic patient cultures with adequate metabolic control compared to the respective normal control group $(\mathrm{P}=0.09$; Figure 3$)$.

\section{Discussion}

Hyperglycemia is considered to have a negative influence on immune-competent cells, a hypothesis supported by the evidence of improvement in the immune-cellular response of diabetic patients after metabolic control (27). However, the insulinopen-



Figure 2. INF- $\gamma$ levels in culture supernatants of peripheral blood mononuclear cells from type 1 and 2 diabetic patients having inadequate (NA) or adequate (A) metabolic control and normal individuals (control 1 (C1) and control 2 (C2) paired by sex and age with type 1 and 2 diabetic patients, respectively). INF- $\gamma$ levels were higher in the A type 1 diabetic group compared to the A type 2 and NA type 1 diabetic groups $(P<0.001$ and 0.009 , respectively; Kruskal-Wallis and Wilcoxon tests). The horizontal lines indicate the median for each group.

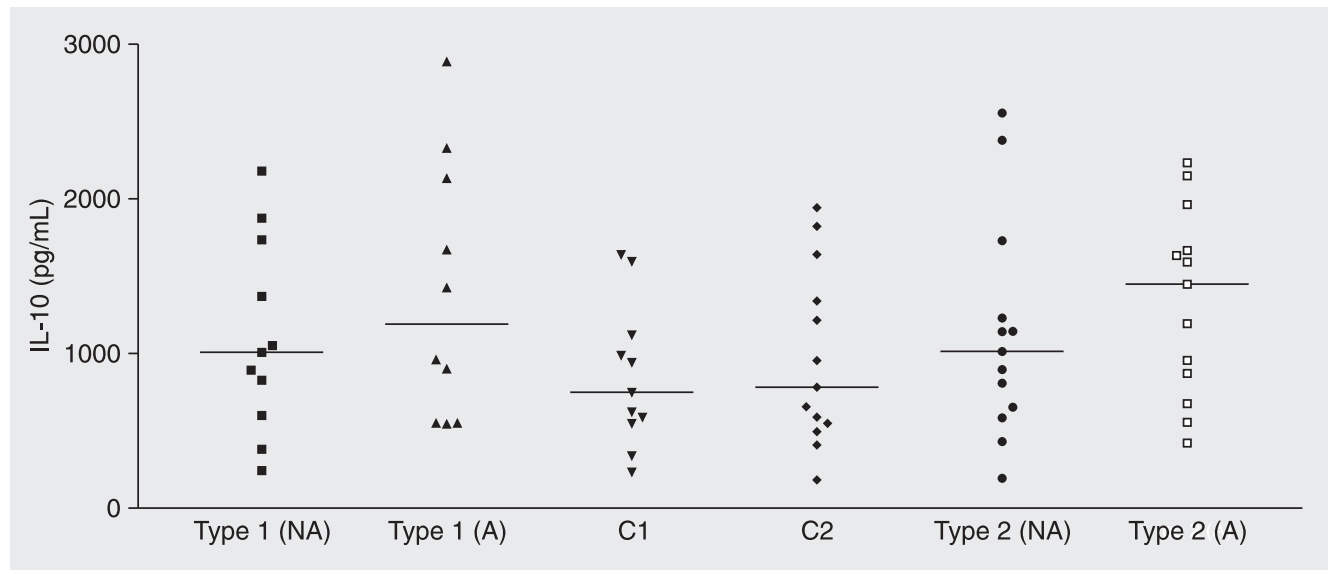

Figure 3. IL-10 levels in culture supernatants of peripheral blood mononuclear cells (PBMC) from type 1 and 2 diabetic patients having inadequate (NA) or adequate (A) metabolic control and normal individuals (control 1 (C1) and control 2 (C2) paired by sex and age with type 1 and 2 diabetic patients, respectively). The increase in IL-10 levels in the PBMC culture supernatants of A type 2 diabetic patients compared to $\mathrm{C} 2$ was not statistically significant $(P=0.09$; Kruskal-Wallis test). The horizontal lines indicate the median for each group. 
ic state may be the reason for these immunological defects (28). Current studies $(29,30)$ suggest an anti-inflammatory effect of insulin even in situations of acute compensation of hyperglycemic metabolic disturbances.

Plasma levels of cytokines in type 1 diabetic patients can be different from those of non-diabetic patients $(31,32)$, but it is well known that the plasma concentrations of cytokines suffer interference from many factors and can be produced by several types of cells. Thus, we used PBMC cultures to evaluate cells of the immunological system, focusing on the susceptibility to infections of these patients.

IFN- $\gamma$ is an important cytokine involved in the immunological defense against intracellular microorganisms which determines a Th1 pattern of the adaptive immune response. According to Faresjö et al. (33), IFN- $\gamma$ levels are increased in the supernatants of PBMC cultures from type 1 diabetic patients during the first month after the diagnosis. In the present investigation, we studied type 1 diabetic patients several years after diagnosis and we observed increased levels of IFN- $\gamma$ in comparison to type 2 diabetic patients. This suggests that the immunological characteristics observed in early stages of this type of diabetes may further persist. Finally, it cannot be excluded that "hyperglycemic memory" confounds the relationship between cell function and concomitant blood glucose concentration, i.e., hyperglycemia-induced changes in gene expression and biological reactions such as advanced glycation-end products in long-lived macromolecules may not be reversed when glycemia returns to normal, so that poor control is progressively "imprinted" on the cells (34), resulting in impairment of cellular immune activity.

Furthermore, metabolic control increased IFN- $\gamma$ concentration in the supernatants of cell cultures from type 1 diabetic patients, showing that an adequate metabolic control may positively influence lymphocyte activ- ity. This increase in lymphocyte activity may have implications not only in the immunological defense against infection but also in the pathological process of type 1 diabetes mellitus, suggesting that adequate metabolic control might stimulate the pancreatic injury in earlier stages of this disease. In contrast, metabolic control did not influence the production of this cytokine in type 2 diabetic patients despite the lower IFN- $\gamma$ levels in the type 2 diabetic groups compared to the normal paired control group. These data may be associated with an immunological defect originating from the chronic disease and the older age of type 2 diabetic patients.

Traditionally, IL-10 is an anti-inflammatory cytokine that can regulate the Th1 pattern of the cellular immune response, but the biological activity of this cytokine seems to be more complex and there is evidence of a pro-inflammatory effect (35-37). In agreement with Geerlings et al. (38), we did not find significant differences in PBMC production of IL-10 between our diabetic and normal individuals, but we observed a tendency to an increase in IL-10 levels in type 2 diabetic patients after they obtained adequate metabolic control.

The increased supernatant levels of IFN$\gamma$ in PBMC cultures from type 1 diabetic patients with adequate metabolic control suggest a pattern of inflammatory response in patients with good metabolic control. Additionally, the tendency to an increase in supernatant IL-10 levels in type 2 diabetic patients with adequate metabolic control suggests that the control of diabetes improves the capacity of activation and maintenance of the immune response, probably diminishing the susceptibility to infections.

\section{Acknowledgments}

The authors are grateful to Maria Aparecida Nunes Ferreira and Sebastião L. Brandão Filho for technical assistance. 


\section{References}

1. Curfs JH, Meis JF, Hoogkamp-Korstanje JA. A primer on cytokines: sources, receptors, effects, and inducers. Clin Microbiol Rev 1997; 10: 742-780.

2. Vlassara $H$, Brownlee $M$, Manogue KR, Dinarello CA, Pasagian A. Cachectin/TNF and IL-1 induced by glucose-modified proteins: role in normal tissue remodeling. Science 1988; 240: 1546-1548.

3. Shanmugam N, Reddy MA, Guha M, Natarajan R. High glucoseinduced expression of proinflammatory cytokine and chemokine genes in monocytic cells. Diabetes 2003; 52: 1256-1264.

4. The effect of intensive treatment of diabetes on the development and progression of long-term complications in insulin-dependent diabetes mellitus. The Diabetes Control and Complications Trial Research Group. N Engl J Med 1993; 329: 977-986.

5. Intensive blood-glucose control with sulphonylureas or insulin compared with conventional treatment and risk of complications in patients with type 2 diabetes (UKPDS 33). UK Prospective Diabetes Study (UKPDS) Group. Lancet 1998; 352: 837-853.

6. Effect of intensive blood-glucose control with metformin on complications in overweight patients with type 2 diabetes (UKPDS 34). UK Prospective Diabetes Study (UKPDS) Group. Lancet 1998; 352: 854-865.

7. Joshi N, Caputo GM, Weitekamp MR, Karchmer AW. Infections in patients with diabetes mellitus. N Engl J Med 1999; 341: 1906-1912.

8. Shah BR, Hux JE. Quantifying the risk of infectious diseases for people with diabetes. Diabetes Care 2003; 26: 510-513.

9. Benfield T, Jensen JS, Nordestgaard BG. Influence of diabetes and hyperglycaemia on infectious disease hospitalisation and outcome. Diabetologia 2007; 50: 549-554.

10. Leibovici L, Yehezkelli Y, Porter A, Regev A, Krauze I, Harell D. Influence of diabetes mellitus and glycaemic control on the characteristics and outcome of common infections. Diabet Med 1996; 13: 457-463.

11. Golden SH, Peart-Vigilance C, Kao WH, Brancati FL. Perioperative glycemic control and the risk of infectious complications in a cohort of adults with diabetes. Diabetes Care 1999; 22: 1408-1414.

12. Tabor CA, Parlette EC. Cutaneous manifestations of diabetes. Signs of poor glycemic control or new-onset disease. Postgrad Med 2006; 119: $38-44$.

13. Szalai G, Fellegi V, Szabo Z, Vitez LC. Mucormycosis mimicks sinusitis in a diabetic adult. Ann N Y Acad Sci 2006; 1084: 520-530.

14. Rodrigues DC, Taba MJ, Novaes AB, Souza SL, Grisi MF. Effect of non-surgical periodontal therapy on glycemic control in patients with type 2 diabetes mellitus. J Periodontol 2003; 74: 1361-1367.

15. Bybee JD, Rogers DE. The phagocytic activity of polymorphonuclear leukocytes obtained from patients with diabetes mellitus. J Lab Clin Med 1964; 64: 1-13.

16. Bagdade JD, Nielson KL, Bulger RJ. Reversible abnormalities in phagocytic function in poorly controlled diabetic patients. Am J Med Sci 1972; 263: 451-456.

17. Stevens DL, Bryant AE, Huffman J, Thompson K, Allen RC. Analysis of circulating phagocyte activity measured by whole blood luminescence: correlations with clinical status. J Infect Dis 1994; 170: 1463-1472.

18. Saeed FA, Castle GE. Neutrophil chemiluminescence during phagocytosis is inhibited by abnormally elevated levels of acetoacetate: implications for diabetic susceptibility to infections. Clin Diagn Lab Immunol 1998; 5: 740-743.

19. Gallacher SJ, Thomson G, Fraser WD, Fisher BM, Gemmell CG,
MacCuish AC. Neutrophil bactericidal function in diabetes mellitus: evidence for association with blood glucose control. Diabet Med 1995; 12: 916-920.

20. Delamaire M, Maugendre D, Moreno M, Le Goff MC, Allannic H, Genetet B. Impaired leucocyte functions in diabetic patients. Diabet Med 1997; 14: 29-34.

21. Dolkart RE, Halpern B, Perlman J. Comparison of antibody responses in normal and alloxan diabetic mice. Diabetes 1971; 20: 162-167.

22. Mahmoud AA, Rodman HM, Mandel MA, Warren KS. Induced and spontaneous diabetes mellitus and suppression of cell-mediated immunologic responses. Granuloma formation, delayed dermal reactivity and allograft rejection. J Clin Invest 1976; 57: 362-367.

23. Foss NT, Polon DP, Takada MH, Foss-Freitas MC, Foss MC. Skin lesions in diabetic patients. Rev Saúde Pública 2005; 39: 677-682.

24. Foss-Freitas MC, Foss NT, Donadi EA, Foss MC. Effect of metabolic control on the in vitro proliferation of peripheral blood mononuclear cells in type 1 and type 2 diabetic patients. São Paulo Med J 2006; 124: 219-222.

25. Foss-Freitas MC, Foss NT, Donadi EA, Foss MC. In vitro TNF-alpha and IL-6 production by adherent peripheral blood mononuclear cells obtained from type 1 and type 2 diabetic patients evaluated according to the metabolic control. Ann NY Acad Sci 2006; 1079: 177-180.

26. Foss NT, de Oliveira EB, Silva CL. Correlation between TNF production, increase of plasma C-reactive protein level and suppression of $\mathrm{T}$ lymphocyte response to concanavalin A during erythema nodosum leprosum. Int $J$ Lepr Other Mycobact Dis 1993; 61: 218226.

27. Geerlings SE, Hoepelman Al. Immune dysfunction in patients with diabetes mellitus (DM). FEMS Immunol Med Microbiol 1999; 26 : 259-265.

28. Moutschen MP, Scheen AJ, Lefebvre PJ. Impaired immune responses in diabetes mellitus: analysis of the factors and mechanisms involved. Relevance to the increased susceptibility of diabetic patients to specific infections. Diabete Metab 1992; 18: 187-201.

29. Stentz FB, Umpierrez GE, Cuervo R, Kitabchi AE. Proinflammatory cytokines, markers of cardiovascular risks, oxidative stress, and lipid peroxidation in patients with hyperglycemic crises. Diabetes 2004; 53: 2079-2086.

30. Dandona P, Aljada A, Mohanty P. The anti-inflammatory and potential anti-atherogenic effect of insulin: a new paradigm. Diabetologia 2002; 45: 924-930.

31. Foss MC, Foss NT, Paccola GM, Silva CL. Serum levels of tumor necrosis factor in insulin-dependent diabetic patients. Braz $J$ Med Biol Res 1992; 25: 239-242.

32. Mysliwska J, Zorena K, Bakowska A, Skuratowicz-Kubica A, Mysliwski A. Significance of tumor necrosis factor alpha in patients with long-standing type-I diabetes mellitus. Horm Metab Res 1998; 30: 158-161.

33. Karlsson Faresjo MG, Ernerudh J, Ludvigsson J. Cytokine profile in children during the first 3 months after the diagnosis of type 1 diabetes. Scand J Immunol 2004; 59: 517-526.

34. Lorenzi M. Glucose toxicity in the vascular complications of diabetes: the cellular perspective. Diabetes Metab Rev 1992; 8: 85-103.

35. Mocellin S, Marincola F, Rossi CR, Nitti D, Lise M. The multifaceted relationship between $\mathrm{LL}-10$ and adaptive immunity: putting together the pieces of a puzzle. Cytokine Growth Factor Rev 2004; 15: 61-76.

36. Langer JA, Cutrone EC, Kotenko S. The class II cytokine receptor 
(CRF2) family: overview and patterns of receptor-ligand interactions. Cytokine Growth Factor Rev 2004; 15: 33-48.

37. Tiittanen M, Huupponen JT, Knip M, Vaarala O. Insulin treatment in patients with type 1 diabetes induces upregulation of regulatory $\mathrm{T}$ cell markers in peripheral blood mononuclear cells stimulated with insulin in vitro. Diabetes 2006; 55: 3446-3454.

38. Geerlings SE, Brouwer EC, Van Kessel KC, Gaastra W, Stolk RP, Hoepelman Al. Cytokine secretion is impaired in women with diabetes mellitus. Eur J Clin Invest 2000; 30: 995-1001. 